\title{
Effects of emotional arousal on multiple memory systems: Evidence from declarative and procedural learning
}

\author{
Stephan Steidl, Salwa Mohi-uddin, and Adam K. Anderson ${ }^{1}$ \\ Department of Psychology, University of Toronto, Toronto, Ontario M5S 3G3, Canada
}

\begin{abstract}
Extensive evidence documents emotional modulation of hippocampus-dependent declarative memory in humans. However, little is known about the emotional modulation of striatum-dependent procedural memory. To address how emotional arousal influences declarative and procedural memory, the current study utilized (1) a picture recognition and (2) a weather prediction (WP) task (a probabilistic classification learning task), which have been shown to rely on hippocampal- and striatum-based memory systems, respectively. Observers viewed arousing or neutral pictures after (Experiment 1) or during (Experiment 2) WP training trials. A 1-wk delayed picture recognition memory test revealed enhanced declarative memory for arousing compared with neutral pictures. Arousal during encoding impaired initial WP acquisition but did not influence retention when tested after a 1-wk delay. Data from a subsequent 3-mo delayed test, however, suggested that arousal during acquisition may enhance remote WP retention. These results suggest a potential dissociation between how readily emotional arousal influences hippocampus-dependent and striatum-dependent memory systems in humans.
\end{abstract}

There is a rich and growing literature demonstrating that emotional arousal enhances declarative memory. This is illustrated, in an example from everyday experience, in the greater retention of a more remote memory of avoiding a disastrous car accident compared with a more recent and mundane memory of where we parked our car earlier in the day. The amygdala appears to serve a critical function in the neurobiology of this phenomenon in both human and nonhuman animals. Numerous studies from nonhuman animal research converge on the appreciation that the amygdala plays a critical role in the emotional modulation of memory retention (for review, see Cahill and McGaugh 1999). In particular, post-encoding manipulation of noradrenergic activation in the amygdala has demonstrated its importance to the differential consolidation of memories associated with sympathetic activation (for review, see McIntyre et al. 2003). In humans, both positron emission tomography (PET) and functional magnetic resonance imaging (fMRI) studies have shown that amygdala activity during encoding is highly correlated with the amount of explicit long-term recall of emotionally arousing material (Cahill et al. 1996; Canli et al. 2000; Dolcos et al. 2004; Kensinger and Schacter 2005). These studies suggest an interaction between the amygdala and a hippocampal-based declarative memory system that supports conscious recollection of past experiences (Packard et al. 1994; Packard and Cahill 2001; Kilpatrick and Cahill 2003). This has been shown directly in a structural equation modeling examination of neuroimaging data, which demonstrates greater amygdala/ipsilateral parahippocampal interactions during emotional relative to neutral event encoding (Kilpatrick and Cahill 2003).

Memory for past events, however, is accomplished via multiple qualitatively and anatomically distinct mechanisms and is thus subserved via multiple memory systems in the brain (Cohen and Squire 1980; Packard et al. 1989; McDonald and White 1993; Schacter and Tulving 1994; Knowlton et al. 1996; White and McDonald 2002). The most strongly established distinction is

\footnotetext{
${ }^{1}$ Corresponding author.
}

E-mail anderson@psych.utoronto.ca; fax (416) 978-4811.

Article is online at http://www.learnmem.org/cgi/doi/10.1101//m.324406. between a hippocampus-based declarative memory system and a striatum-based nondeclarative/procedural memory system (Cohen and Squire 1980), with the latter supporting forms of memory independently of the conscious experience of remembering. In nonhuman animals, the amygdala has been shown to modulate memory processes subserved by both declarative and procedural memory systems (Packard et al. 1994; Packard and Cahill 2001; McGaugh et al. 2002; McGaugh 2004). For example, one study in rats has shown that the amygdala can interact with, and enhance, both the hippocampus-based memory system and the striatum-based procedural memory system (Packard et al. 1994). Post-training intra-amygdala injection of the catecholaminergic agonist $d$-amphetamine was shown to enhance retention on separate versions of a spatial, hippocampus-dependent water maze task and a cued, caudate nucleus-dependent water maze task. This suggests that the amygdala plays a pervasive neuromodulatory role in enhancing memory formation in multiple memory systems. Thus, in humans, amygdala modulation of memory for emotionally arousing events may be supported by a parallel enhancement of phenomenologically and anatomically distinct forms of memory. In our example of circumventing a near devastating accident, recollection for the event may be enhanced and, in parallel, so may be the actions that were taken to navigate it successfully. However, whether arousal and/or the amygdala can modulate striatum-based memory in humans to the same extent as it has been shown to affect hippocampusbased memory is not yet known.

In the current study, the weather prediction (WP) task, a striatum-dependant learning task (Knowlton et al. 1994), was used to study the effects of emotional arousal on implicit/ procedural memory. The WP task is a probabilistic classification task that initially depends upon implicit learning of complex cue-outcome contingencies. Consistent with its reliance on procedural striatal versus declarative hippocampal learning, early phases of the acquisition of the WP task recruit the striatum (Poldrack et al. 1999). Initial phases of WP acquisition are intact in medial temporal lobe amnesiacs but disrupted in patients with Parkinson's disease (Knowlton et al. 1994). Amygdala enhancement of declarative memory in humans has been shown to de- 
pend on the degree of arousal evoked during encoding of emotionally significant pictures (Hamann et al. 1999; Canli et al. 2000). Combining these two lines of evidence, emotional arousal evoked by emotionally provocative pictures and thereby associated amygdala activation, may enhance WP acquisition and consolidation.

The present experiments were designed to examine how emotional arousal influences memory traces in humans that lead to recollective experience of the learning context, and forms of memory that support implicit based procedural learning. Arousal was manipulated by presenting subjects with emotionally positively or negatively arousing and/or neutral pictures at regular intervals after (Experiment 1) or during (Experiment 2) the WP task. Subjects participated in two WP task sessions $1 \mathrm{wk}$ apart to examine the effects of emotional arousal during encoding on acquisition and subsequent consolidation of procedural memory. A 1-wk delayed picture recognition memory task was administered to the same subjects, allowing for comparison of the effects of emotional arousal on striatal-dependent implicit learning and hippocampus-dependent explicit memory for the learning context.

\section{Results}

\section{Experiment 1: Post-encoding manipulation of arousal}

In Experiment 1 , emotionally positively or negatively arousing and/or neutral pictures were presented following WP acquisition to examine the effects of arousal on consolidation independently of its influence on encoding, consistent with the nonhuman animal models of arousal enhancement of declarative and procedural memory (Packard et al. 1994).

\section{Arousal ratings}

Ratings data from the picture viewing block were submitted to a one-way ANOVA with condition (arousal and neutral) submitted as a between subject factor. The arousal condition (collapsed across negative, positive, and neutral picture types) was associated with an increase in self-reported arousal relative to the neutral condition, $F_{(1,40)}=36.87, P<0.0001$ (Fig. 1A).

\section{Recognition task}

As illustrated in Figure 1B, paralleling the arousal ratings, mean corrected picture recognition during session 2 depended on encoding conditions, demonstrating enhanced memory for arousing picture contexts. Overall corrected recognition memory $1 \mathrm{wk}$ later was significantly enhanced in the arousal (collapsed across negative, positive, and neutral picture types) relative to the neutral condition, $F_{(1,40)}=8.51, P<0.006$. Specifically, arousing pictures in the arousing condition were better remembered than were neutral pictures in the neutral condition, $F_{(1,40)}=18.51$, $P<0.0001$. Neutral items in the arousal condition tended to be better remembered than were those in the neutral condition $(33.3 \% \mathrm{SE}=5.0$ vs. $24.2 \% \mathrm{SE}=4.0)$, but this did not reach statistical significance, $F_{(1,40)}=1.99, P>0.17$.

\section{Weather prediction}

\section{Session 1}

Consistent with successful acquisition, participants showed significant WP learning across trial blocks, $F_{(4,160)}=10.23$, $P<0.0001$, with a linear contrast revealing a strong increase from block 1 through $5, F_{(4,160)}=32.63, P<0.0001$. Neither overall performance, $F_{(1,40)}=1.05, P>0.31$ (Fig. 1C), nor rate of learning, $F<1$, differed depending on post-encoding condition (Fig. 1D).
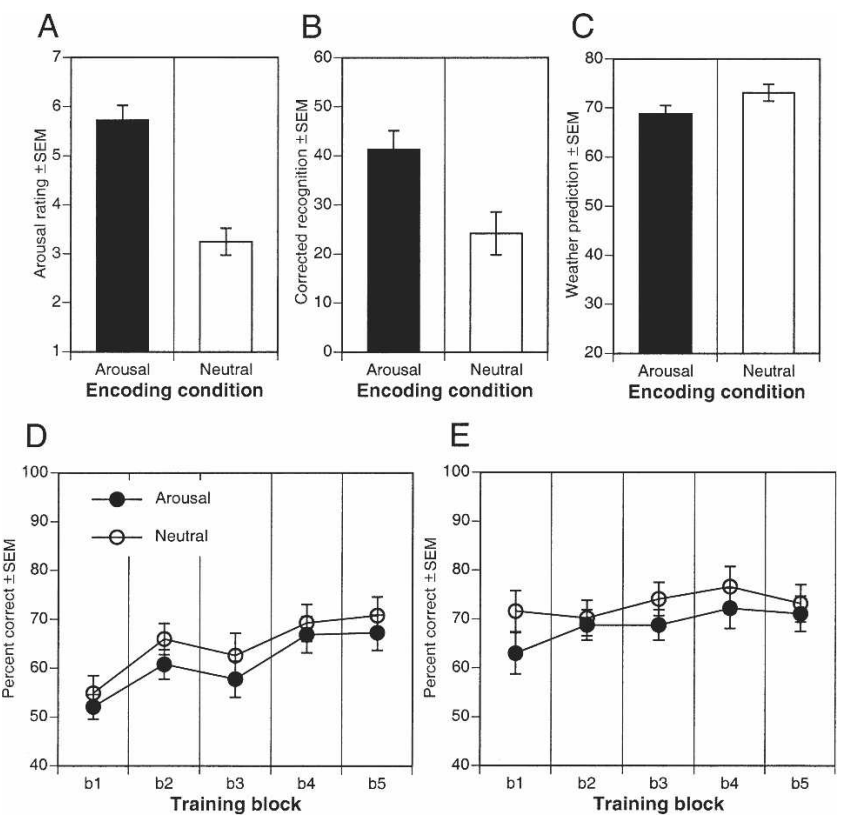

Figure 1. Relation between post-encoding manipulation of arousal and WP learning in Experiment 1. (A) Overall self-reported arousal to arousing and neutral pictures presented following initial WP acquisition. (B) Oneweek delayed recognition memory for pictures presented following WP acquisition. (C) Overall WP initial acquisition in each post-encoding condition. (D) WP acquisition curves for session 1. (E) Retention and new WP acquisition curves for session 2 given $7 \mathrm{~d}$ later. Arousal and neutral refer to initial encoding conditions.

\section{Session 2}

Arousal level did not significantly enhance consolidation of WP learning (Fig. 1E). We first assessed the presence of enhanced consolidation of WP learning by comparing the last training block (block 5) from session 1 with the first training block (block 1 ) from session 2 . Both groups showed retention of skills learned over the 1-wk period separating session 1 and session 2 , with no significant drop off in performance, $F<1$. There was no evidence of an interaction with encoding condition, $F<1$, suggesting that the level of retention across the 1-wk period was not influenced by emotional arousal experienced following initial training in session 1.

To assess whether arousal encoding conditions would enhance further learning of WP cue-outcome associations, we examined performance with additional training. A main effect of block, $F_{(4,160)}=2.96, P<0.03$, indicated further significant increases in performance with additional training trials during session 2, with a significant linear contrast across blocks, $F_{(1,160)}=8.77, P<0.004$ (Fig. 1E). However, as during initial training, there was no evidence to suggest that overall performance was enhanced in the post-encoding arousal condition, $F<1$, nor was there any evidence of an interaction between trial block and encoding condition, $F<1$, suggesting that the further acquisition of cue-outcome relations across blocks of trials was not modulated by level of arousal following initial encoding.

To ensure that the failure of post-encoding arousal to enhance further acquisition did not reflect a ceiling effect, we performed a median split analysis. In each of the arousal and neutral conditions, the bottom half performing subjects on block 1 of session 2 were included in an additional analysis. Analysis across days revealed that these individuals demonstrated significant forgetting from block 5 of session $1, F_{(1,18)}=6.10, P<0.03$, but there was no evidence of differential forgetting between arousal conditions, $F<1$. These individuals demonstrated robust learn- 
ing across blocks with additional training during session 2, $F_{(1,72)}=15.44, P<0.0002$, but there was no evidence that postencoding arousal condition altered this subsequent learning, $F<1$. A similar median split analysis was performed on accuracy at the end of session 1 acquisition (block 5) to identify participants that were below ceiling level performance and thus may be most susceptible to enhanced learning with post-encoding arousal. There was no evidence of post-encoding arousal on retention between sessions (session 2 block 1 ), $F<1$, or enhancement of further learning across blocks during session $2, F<1$.

\section{Transfer task}

As illustrated in Figure 2, A and B, consistent with probability matching, the percentage of sunshine estimation increased with increasing cue-outcome predictability; however, arousal following encoding did not enhance this probability estimation.

\section{Session 1}

Estimation of sunshine depended upon cue-outcome predictability, $F_{(4,160)}=13.97, P<0.0001$, being linearly associated with increasing probability of sunshine, $F_{(1,160)}=54.69, P<0.0001$. Overall, participants tended to overestimate the least predictive and underestimate the most predictive events, regardless of encoding condition.

\section{Session 2}

Estimation of sunshine again depended upon cue-outcome predictability, $F_{(4,160)}=18.65, P<0.0001$; however, there was no effect of condition or an interaction with condition, suggesting that manipulation of arousal following initial encoding did not influence the estimation of cue-outcome probability, $F<1$.

\section{Remote retrieval}

\section{Session 3}

To examine the long-term effects of post-encoding arousal on WP learning, participants were asked to come back for an additional test session $\sim 3$ mo following initial encoding. These analyses are considered exploratory as they are based on more limited observations from participants who partook in the final test session. WP performance data were submitted to an additional ANOVA with encoding condition (arousal and neutral) as a between-subjects variable and session (session 1 and session 3), and training block ( 1 through 5 ) as within-subjects variables. Collapsing across sessions, there was significant learning across trial blocks, $F_{(4,84)}=7.31, P<0.0001$. Learning across trial blocks interacted with session, being more pronounced for session 1 , $F_{(4,84)}=8.75, P<0.0001$. There was a significant effect of session, $F_{(1,21)}=6.00, P<0.03$, revealing that performance even after a $\sim 3$-mo delay was enhanced relative to initial learning. However,
A

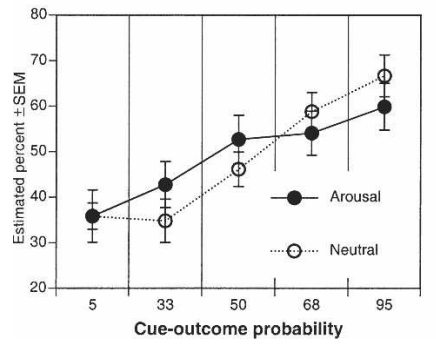

\section{B}

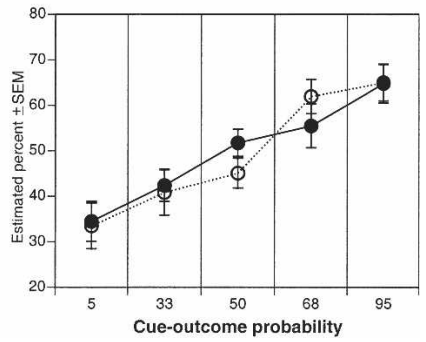

Figure 2. Probability matching in learning of cue-outcome probabilities in Experiment 1 on session $1(A)$ and retention and further learning during session $2(B)$ in the arousal and neutral post-encoding conditions. Arousal and neutral refer to post-encoding conditions for session 1. there was no evidence of a main effect or an interaction with post-encoding condition, all $P>0.32$.

To more closely examine if forgetting occurred after the 3-mo delay, a focused analysis of performance at the end of session 2 (block 5) was compared directly with the beginning of session 3 (block 1) across the two encoding conditions. A main effect of delay revealed significant forgetting $(77.3, \mathrm{SE}=2.9$ vs. $69.2, \mathrm{SE}=3.7), F_{(1,21)}=4.71, P<0.05$. However, the rate of this forgetting did not differ depending on post-encoding condition, $F<1$.

\section{Conclusions}

All participants showed significant learning during both sessions of the WP task, and observed performance parallels acquisition rates seen in other studies using the WP task (Knowlton et al. 1996). Furthermore, participants showed retention of learning across the 1-wk delay separating WP sessions. Post-encoding arousal manipulations did not affect consolidation of learning occurring during the first WP session, nor did it affect the rates of additional acquisition during session 2 . The lack of effect of postencoding arousal on implicit memory was not due to a ceiling effect in participant performance as participants clearly showed continuing improvements in performance across sessions. Furthermore, those subjects with the poorest performance levels at the end of day 1 were not any more susceptible to further enhancement in WP acquisition, regardless of arousal condition. Performance on the transfer task revealed that participants correctly gauged relative cue-outcome contingencies but did not appear to show any significant increase in probability matching performance across days, regardless of encoding condition.

By contrast, 1-wk delayed recognition memory was significantly enhanced for the arousing relative to neutral condition, indicating that arousal enhanced declarative memory for the episodic context following WP learning.

\section{Results}

Experiment 2: Manipulation of arousal during encoding Evidence suggests that post-encoding arousal interacts with the amount of arousal experienced during encoding. In particular, post-training arousal enhances memory for items that induced the most arousal during training (Cahill and Alkire 2003; Cahill et al. 2003). The observed lack of enhancement due to postencoding arousal in Experiment 1 may be explained by the fact that the performance of the WP task (i.e., predicting sunshine from combinations of geometric patterns) was not emotionally arousing, and thus sufficient arousal may not have been evoked during encoding. Experiment 2 examined the effect of arousal evoked during WP learning.

\section{Arousal ratings}

The arousal condition (collapsed across negative, positive, and neutral picture types) was associated with an increase in selfreported arousal relative to the neutral condition, $F_{(1,40)}=25.58$, $P<0.0001$ (Fig. 3A).

\section{Recognition task}

As illustrated in Figure 3B, mean corrected picture recognition (hits-false alarms) significantly differed across encoding conditions. Recognition memory was significantly more accurate in the arousing relative to neutral condition, $F_{(1,48)}=12.09$, $P<0.002$. Specifically, arousing pictures in the arousing condition were better remembered than were neutral pictures in the neutral condition, $F_{(1,48)}=22.63, P<0.0001$, and this enhanced memory extended to neutral pictures in the arousal condition being remembered more accurately than were neutral pictures in

\section{Learning \& Memory


A

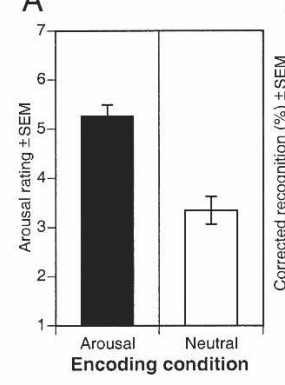

B
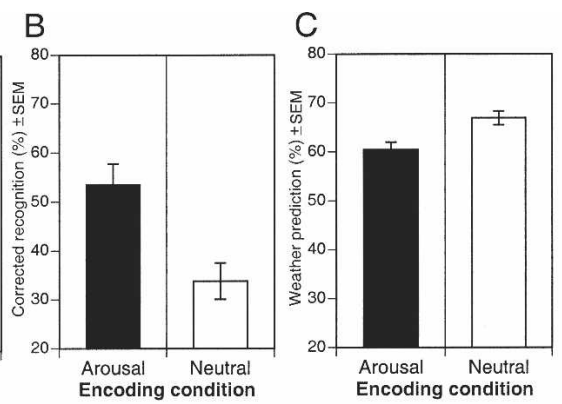

D

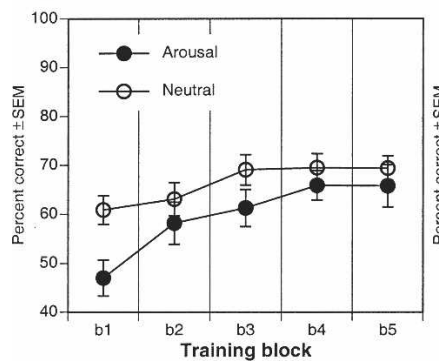

E

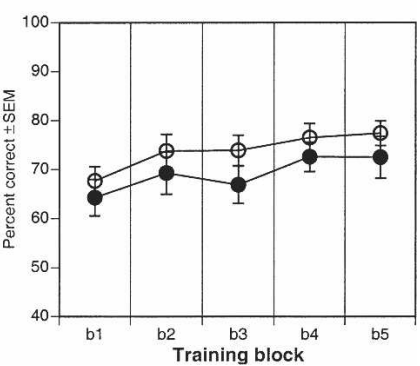

Figure 3. Relation between manipulation of arousal during encoding and WP learning in Experiment 2. (A) Overall self-reported arousal to arousing and neutral pictures during initial WP acquisition. (B) One-week delayed recognition memory for pictures presented during WP acquisition. (C) Overall WP initial acquisition in each encoding condition. (D) WP acquisition curves for session 1. (E) Retention and new WP acquisition curves for session 2 given $7 \mathrm{~d}$ later. Arousal and neutral refer to initial encoding conditions.

the neutral condition $(48.8 \% \mathrm{SE}=6.3$ vs. $33.8 \% \mathrm{SE}=3.7)$, $F_{(1,48)}=4.20, P<0.05$.

\section{Weather prediction}

\section{Session 1}

As illustrated in Figure 3, C and D, arousal level during encoding did not significantly enhance WP acquisition. Consistent with successful acquisition, observers showed significant learning across trial blocks, $F_{(1,192)}=11.91, P<0.0001$, increasing linearly from block 1 through 5, $F_{(1,192)}=39.92, P<0.0001$. There was overall lower performance in the arousal condition compared with the neutral condition, $F_{(1,48)}=4.16, P<0.05$. There was no significant interaction between encoding arousal and training block, $F_{(1,192)}=1.33, P>0.26$, suggesting that the rate of acquisition of cue-outcome relations across blocks of trials was not enhanced by intermittent emotional arousal. There was, however, indication of an initial acquisition impairment in the arousal condition. A simple effects analysis revealed that performance in the arousal condition during initial acquisition in block 1 was significantly below that of the neutral condition, $F_{(1,48)}=9.03, P<0.005$.

\section{Session 2}

As illustrated in Figure 3E, arousal level during initial WP acquisition did not significantly influence WP retention. We first assessed the presence of enhanced consolidation of WP learning by comparing block 5 of session 1 with block 1 of session 2. Both groups showed retention of WP learning over the 1-wk period separating WP sessions, with no significant drop off in performance, $F<1$. There was no evidence of an interaction with encoding condition, $F<1$, suggesting that performance across the 1 -wk period was not influenced by emotional arousal experienced during initial training.
To assess whether arousal encoding conditions would enhance further learning of WP cue-outcome associations, we examined performance with additional training. A main effect of block, $F_{(4,192)}=6.88, P<0.0001$, indicated that participants in both conditions demonstrated further significant increases in performance with additional training trials during session 2 , linearly increasing from block 1 to block $5, F_{(1,192)}=22.31$, $P<0.0001$. However, as during initial training, there was no evidence to suggest that overall performance was greater in the arousal condition, $F<1$, nor was there any evidence of an interaction between trial block and encoding condition, $F<1$, suggesting that the further acquisition of cue-outcome relations across blocks of trials was not modulated by level of arousal during initial encoding.

\section{Transfer task}

As illustrated in Figure 4, A and B, consistent with probability matching, the percentage of sunshine estimation increased with increasing cue-outcome predictability; however, arousal during encoding did not enhance this probability estimation.

Session 1

Estimation of sunshine depended upon cue-outcome predictability, $F_{(4,192)}=21.30, P<0.0001$, linearly associated with increasing predictability of sunshine, $F_{(1,192)}=84.60, P<0.0001$. Overall, participants tended to overestimate the least predictive and underestimate the most predictive events, regardless of encoding condition. There was no effect of encoding condition, $F<1$.

Session 2

Estimation of sunshine depended upon cue-outcome predictability, $F_{(4,192)}=34.01, P<0.0001$, linearly associated with increasing predictability of sunshine, $F_{(1,192)}=134.77, P<0.0001$. There was no effect of encoding condition, $F<1$.

\section{Remote retrieval}

Session 3

To examine the long-term effects of arousal during encoding on WP learning, participants were asked to return for an additional test session $\sim 3$ mo following initial encoding (arousal condition, average $=125 \mathrm{~d}$ [range 112-131]; neutral condition, average $=110 \mathrm{~d}$ [range 104-121]). These analyses are considered exploratory as they are based on limited observations from participants who partook in a final test session (arousal $N=5$; neutral $N=5$ ).

\section{Picture recognition}

Although, picture recognition was not assayed again in the third session, we analyzed recognition data from session 2 to determine if there was sufficient power to demonstrate differences in
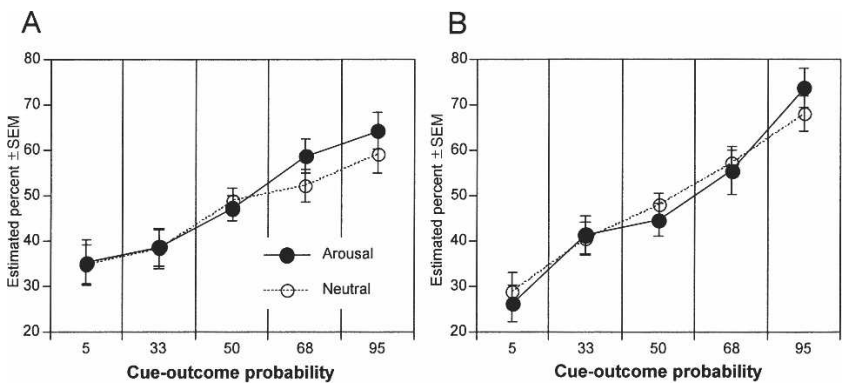

Figure 4. Probability matching in learning of cue-outcome probabilities in Experiment 2 on session $1(A)$ and retention and further learning during session $2(B)$ in the arousal and neutral initial encoding conditions. Arousal and neutral refer to encoding conditions for session 1. 
declarative memory from the smaller sample. Indeed, there was a robust enhancement of recognition for arousing pictures in the arousal condition relative to neutral pictures in the neutral condition $(57.5 \% \mathrm{SE}=4.9$ vs. $30.4 \% \mathrm{SE}=3.7), F_{(1,8)}=19.79$, $P<0.003$. In the arousing condition, memory for neutral and arousing pictures was statistically equivalent $(57.5 \% \mathrm{SE}=4.9$ vs. $56.0 \% \mathrm{SE}=15.4), F<1$.

\section{WP performance}

Data were submitted to an additional ANOVA with encoding condition (arousal and neutral) as a between-subjects variable and session (session 1 and session 3 ), and training block (1 through 5) as within-subjects variables. WP performance increased across training blocks, $F_{(1,32)}=2.80, P<0.05$, and across repeated training sessions, $F_{(1,8)}=45.47, P<0.0001$. Critically, there was a significant condition by session interaction, $F_{(1,8)}=9.28, P<0.02$. Whereas WP performance was better under initial neutral training conditions on session 1 , after substantial delay in session 3, WP performance was now enhanced for initial arousing relative to neutral encoding conditions. A three-way interaction, among condition, session, and block, $F_{(4,32)}=2.85$, $P<0.04$, revealed that this delayed arousal enhancement was most apparent from the end of session 1 to the beginning of session 3. A focused analysis of this higher-order interaction (block 5 of session 1 and block 1 of session 3) was significant, $F_{(1,8)}=6.90, P<0.03$. Whereas WP performance under neutral encoding conditions was subject to forgetting across the ensuing 3-mo delay, arousal during initial encoding resulted in greater performance at remote test relative to initial performance. An analysis of performance changes from session 2 to session 3 confirmed this overall pattern, with significant forgetting occurring over the 3-mo delay in the neutral condition, $F_{(1,4)}=7.54$, $P=0.05$, and a nonsignificant increase in performance in the arousal condition $F<1$ (Fig. 5).

\section{Conclusions}

One-week delayed recognition memory for picture contexts, in which the WP task was acquired, was significantly enhanced for the arousing relative to neutral condition. On the other hand, induction of emotional arousal during encoding of the WP task was not associated with enhanced rates of acquisition, nor was it associated with enhanced consolidation of learning following a 1-wk delay. There was evidence, however, that arousal during encoding impaired initial WP learning. This initial impairment was associated with a long-term enhancement of WP learning. Remote memory, $\sim 3$ mo after initial encoding, was significantly

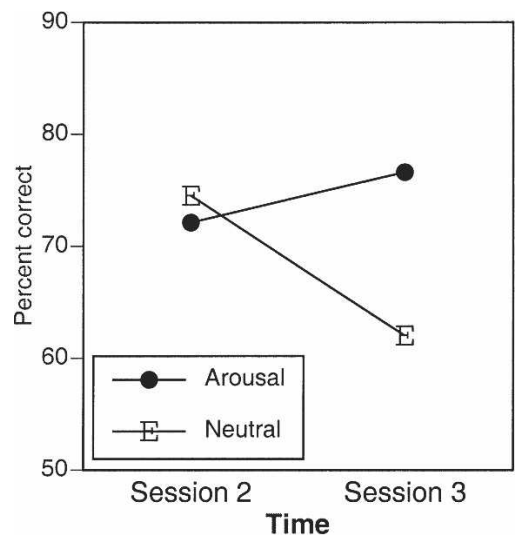

Figure 5. Remote retention of weather prediction learning. Performance is plotted from session 2 to $\sim 3$ mo later during session 3 of testing. Arousal and neutral refer to initial encoding conditions. enhanced for participants in the arousal condition. These differences reflect differential rates of forgetting, as indicated by a significant decrease in WP retention after a 3-mo delay in the neutral but not the arousal encoding conditions.

\section{Discussion}

Emotional arousal was associated with robust enhancement of explicit recognition of the WP learning context, as indexed by enhanced memory for the pictures presented in the arousing relative to the neutral conditions. By contrast, arousal after (Experiment 1) or during (Experiment 2) encoding did not enhance implicit probabilistic classification learning, as indexed by WP performance. Arousal also did not enhance retention of WP learning as indexed by a 1-wk delayed test, nor did it alter the rate of additional acquisition of WP learning 1 wk later. Arousal during encoding was, however, associated with an initial impairment in performance. This impaired expression of learning was associated with later enhanced WP retention on a remote memory test 3 mo following initial learning. This long-term enhancement may be ascribed to influences on either procedural/ implicit or declarative/explicit learning. The observed dissociation between the susceptibility of declarative and procedural forms of memory to emotional enhancement suggests that arousal may have a more potent influence on hippocampal based memory systems that support explicit recollection than on striatal-based implicit learning.

Arousal during encoding appeared to have had a retarding effect on initial WP acquisition within the first 30 trials of exposure to the cue-outcome contingencies. This initial impairing effect may have been due to the distracting influence of highly arousing picture content (see MacKay et al. 2004; Anderson 2005; Schimmack and Derryberry 2005). In addition, it could represent altered consolidation of implicit learning. The failure to demonstrate enhanced acquisition may be contrasted with an augmentation of later retrieval of these memory traces, consistent with the proposed influence of arousal on memory consolidation (McGaugh 2004). Studies of the effects of arousal on explicit memory have shown that arousing study conditions often result in an initial impairment in immediate retrieval (Kleinsmith and Kaplan, 1963). Walker and Tarte's (1963) "action-decrement" theory suggests this reflects a temporary inaccessibility to memory traces whose consolidation has been augmented. Since the WP task involves simultaneous retrieval and consolidation, arousing conditions may have indeed altered consolidation during initial acquisition, impairing the capacity to retrieve implicitly learned associations. This immediate impairment in explicit memory has often been accompanied by reminiscence-enhanced memory at delayed relative to initial test (Kleinsmith and Kaplan 1963). Similarly, Okuda et al. (2004) have shown in rats that had experienced training-associated arousal, additional post-training arousal induced by corticosterone-impaired object recognition performance when tested $1 \mathrm{~h}$ after testing, while enhancing object recognition performance when tested at $24 \mathrm{~h}$, suggesting that the effect of arousal varies across time. In the current studies, although greater WP memory was not found 1 wk following acquisition, our exploratory analyses of performance after a 3-mo delay revealed a pattern quite similar to that found with explicit memory, with the arousal manipulation producing initial impaired memory and later enhanced retention. This pattern was unlikely due to regression toward the mean, as WP performance was in fact impaired at the beginning of acquisition (session 1) but greater than neutral encoding conditions after a 3-mo delay.

Different tests of implicit memory have been shown to have different rates of forgetting (Schacter 1987); thus, the importance of the 3-mo delayed test may be due to the relative resistance of

\section{Learning \& Memory}

www.learnmem.org 
WP memory to decay (Fig. 5). After a 1-wk delay, there was no evidence of forgetting on WP acquisition in either encoding condition, unlike the robust forgetting found in studies of explicit memory for arousing and neutral events (see Kleinsmith and Kaplan 1963; LaBar and Phelps 1998; Anderson et al. 2006). Given that the effects of emotional arousal are often observed through altering the rate of memory decay, the resistance of WP learning to decay complicates investigations of arousal modulation of procedural learning. As such, the strongest test of arousal on procedural skill learning, as indexed by the WP task, would be after significant forgetting has occurred, at much longer intervals than are typically used in testing long-term declarative memory. We provide preliminary evidence from a remote test of decreased WP retention under neutral encoding conditions (i.e., significant forgetting occurred), and relative maintenance under arousal encoding conditions (i.e., no evidence of forgetting). However, due to the small participating samples, these data must be considered motivation for future testing at remote delays.

In contrast with the effects of arousal during encoding, the present studies showed no effect of post-encoding arousal manipulations. In animal models that use motivated learning (e.g., shock or food), post-training arousal enhances memory for events associated with emotional significance, as in passive avoidance learning, where memory is tested for locations previously associated with shock. Studies examining this notion directly have shown the importance of encoding arousal level for post-encoding arousal augmentation of memory consolidation (Okuda et al. 2004). This interaction between encoding and postencoding arousal may help explain the lack of post-training arousal enhancement obtained in the present study. In humans, although post-encoding arousal can enhance memory for prior presentations of nonarousing events (Nielson et al. 2005; Anderson et al. 2006), this enhancement has been shown to be most prominent for more arousing events (Cahill and Alkire 2003; Cahill et al. 2003). For example, it has been shown that posttraining administration of either epinephrine (Cahill and Alkire 2003) or cold pressor stress (Cahill et al. 2003) enhances longterm memory ( $1 \mathrm{wk}$ ) for arousing material encountered during the training episode but not for nonarousing material. This may indicate why arousal during, but not after encoding, may have successfully modulated remote memory for the WP task. It is likely that the self-reported arousal evoked during WP learning in Experiment 2 had a trajectory that continued following encoding, as well. Thus, subjects in Experiment 2, but not Experiment 1 , may have experienced increased arousal during and following encoding, conditions that may have permitted the enhancement of long-term memory traces (Cahill and Alkire 2003; Cahill et al. 2003; Okuda et al. 2004) supporting both procedural memory and its episodic context.

Enhancement of procedural memory may also be related to the particular type of arousal evoked. Memory enhancement associated with viewing emotionally arousing slides is blocked by systemic $\beta$-adrenergic antagonism, suggesting that these memory enhancements are associated with $\beta$-adrenergic activation (Cahill et al. 1994). On the other hand, cold pressor stress induces robust cortisol activation (Lovallo 1975). Administration of either epinephrine (Cahill and Alkire 2003) or cold pressor stress (Cahill et al. 2003) has been shown to enhance declarative memory for arousing material, but the effect of neither type of arousal on procedural memory has previously been tested. While the current experiments show that procedural memory may be less prominently affected by emotionally arousing pictures, it cannot be ruled out that adrenocortical-based arousal (e.g., evoked by cold pressor stress) can result in more pronounced enhancement of procedural memory.

A growing body of literature documents gender differences in the effects of emotional arousal on memory (Cahill 2003). For example, imaging studies consistently indicate involvement of the left amygdala in memory for emotional material for females but an involvement of the right amygdala in memory for the same material in males (Canli et al. 2002; Cahill et al. 2004). While this warrants more detailed analysis, the disproportionate number of female subjects used in the current studies does not allow for any conclusions about whether potential genderrelated differences in the arousal modulation of declarative memory extends to procedural memory as well.

It is unclear to what extent learning in the current experiment relied exclusively on implicit memory. Previous studies have suggested a change in underlying memory systems used in the WP task with increasing training, such that early on during training learning depends on an intact striatum, while later on during training, learning depends on an intact hippocampus (Reber et al. 1996). Functional imaging studies have supported this switch in engaged memory systems, showing activation of the striatum followed by later activation of the hippocampus (Poldrack et al. 1999; Iaria et al. 2003). In the current study, the amount of trials used in each of two sessions should have ultimately induced recruitment of both memory systems, with later performance being supported more by declarative knowledge. With respect to earlier implicit contributions from procedural memory, the initial impairment in acquisition found in Experiment 2 might reflect a disruption of implicit memory consolidation, which we found was associated with enhanced remote WP retention. However, the present results cannot disentangle whether this pattern of early impairment and later enhancement reflects the working of enhanced procedural or declarative memory for the WP task following a 3-mo delay. Evidence from probability matching in the transfer task reveals that even on cue-outcome contingencies of $>95 \%$ diagnosticity, where declarative memory would likely have its greatest influence, verbal estimates were on average $65 \%$. The data suggest that performance on the WP task may have relied more heavily on the stochastic and likely implicit nature of task learning, and less on explicit declarative associations as suggested by prior studies with the WP task. Unlike prior examinations, arousal manipulations used in the current studies may have interfered with the proposed switch in underlying memory systems. Alternatively, the interspersed picture events may have served to impair declarative memory. Thus, with behavioral indices alone it is not possible to determine whether or not a switch occurred in the relative contributions of memory systems in the present studies.

A detailed analysis of WP performance has shown that at least three different strategies can be used to learn the WP task (Gluck et al. 2002). First, a singleton strategy involves subjects learning the correct outcomes associated with single cards (i.e., memorizing the single cards). Second, a one-cue learning strategy involves subjects basing their responses on the presence/absence of a single card. Third, a multicue learning strategy involves subjects basing their responses on combinations of cue cards present. Furthermore, use of each of these strategies changes across WP trials, such that performance is best described by a singleton strategy early on while being more consistent with a multicue strategy later in training, which may mirror the switch from striatal to hippocampal systems. However, it is unclear to what extent each of these strategies can be learned in a nondeclarative or declarative manner, and subjects may in fact be using combinations of strategies (Gluck et al. 2002). As such it may be the case that different strategies (and associated memory systems) are more or less prone to emotional arousal effects. However, strategies were not assessed in the present experiments and future studies might benefit from examining arousal modulation of different strategies for procedural learning. 
By replicating prior studies, enhancement of explicit memory by emotional arousal suggests that degree of arousal and associated amygdala related activity (Cahill et al. 1996; Canli et al. 2000; Dolcos et al. 2004; Kensinger and Schacter 2005) was sufficient to enhance memory for the recollective context in which the WP task was performed. In contrast, this influence may not be as pronounced for the procedural learning taking place in arousing contexts. Enhanced declarative memory for emotionally arousing events may reflect that these events are intrinsically distinctive and memorable, and that memory is tested for the events themselves that evoke arousal. In addition to enhanced memory for events that were intrinsically emotionally arousing, a summary analysis across the two experiments revealed that neutral pictures in the arousing condition were better remembered than were neutral events in the neutral condition, $F_{(1,90)}=5.87, P<0.02$. Although this finding is in part complicated by the rarity of presentation of neutral events in the arousal conditions, this pattern is consistent with other studies showing enhanced declarative memory for neutral indistinct events presented in arousing contexts, evoked through physical exertion (see Nielson et al. 1996) or through presentation of arousing pictures, as used here (see Anderson et al. 2006). Thus, we suggest that hippocampal-based episodic/declarative memory traces supporting recollection of temporal and spatial context are more malleable and thus more readily influenced by induction of systemic arousal than are procedural memory traces.

In summary, arousal during encoding impaired initial WP acquisition but did not influence retention when tested after a 1-wk delay. By contrast, arousal enhanced 1-wk delayed recognition memory for the picture contexts in which the WP task was performed. A subsequent 3-mo delayed test, however, suggested that arousal during acquisition may enhance remote WP retention. These results suggest a potential dissociation between the influence of arousal on distinct memory systems in humanswith emotional arousal more readily modulating hippocampusdependent than striatum-dependent memory.

\section{Materials and Methods}

\section{Experiment 1}

\section{Participants}

Subjects were recruited through advertisements posted throughout the University of Toronto campus as well as through introductory psychology classes. A total of 42 subjects (age range $=18-43 \mathrm{yr}$ ) participated in the study which involved two sessions separated by $\sim 1 \mathrm{wk}$. Subjects were randomly assigned to either the arousal condition $(n=21$; nine males, 12 females) or the neutral condition ( $n=21$; six males, 15 females).

\section{Materials, design, and procedure}

\section{The WP task}

Participants performed the WP task on a computer (adapted from the method of Knowlton et al. 1996). The WP task was designed by using Super Lab (Version 2.0, Cedrus Inc) and viewed through a 17-inch flat-screen LCD monitor. Participants were presented with varying combinations of four types of cues depicting simple geometric forms: seven black squares, 10 black triangles, nine black circles, and 13 black diamonds. On each trial, combinations of one, two, or three of these cues were presented side by side on the computer screen (14 possible cue combinations) (Table 1), and individuals were asked to predict the weather outcome as either sunshine or rain by pressing one of two keys indicated on a keyboard. Each of the four cards was associated with an outcome of rain or sunshine with a fixed probability. Feedback was given after each response to indicate a correct or incorrect response. In the event of a correct response, subjects heard a highpitch sound and were shown a happy face as well as a text mes-
Table 1. Weather prediction task probability structure ${ }^{a}$

\begin{tabular}{lccccc}
\hline Cue no. & $\begin{array}{c}\text { Cue } \\
\text { arrangement }\end{array}$ & Frequency & Sun & Rain & $\begin{array}{c}P \\
\text { (Sunshine) }\end{array}$ \\
\hline 1 & 1100 & 4 & 4 & 0 & 1 \\
2 & 1110 & 1 & 1 & 0 & 1 \\
3 & 1000 & 7 & 6 & 1 & 0.86 \\
4 & 1010 & 4 & 3 & 1 & 0.75 \\
5 & 0100 & 5 & 3 & 2 & 0.60 \\
6 & 1001 & 2 & 1 & 1 & 0.50 \\
7 & 0110 & 2 & 1 & 1 & 0.50 \\
8 & 1011 & 2 & 1 & 1 & 0.50 \\
9 & 1101 & 2 & 1 & 1 & 0.50 \\
10 & 0010 & 5 & 2 & 3 & 0.40 \\
11 & 0101 & 4 & 1 & 3 & 0.25 \\
12 & 0001 & 7 & 1 & 6 & 0.14 \\
13 & 0111 & 1 & 0 & 1 & 0 \\
14 & 0011 & 4 & 0 & 4 & 0 \\
\hline
\end{tabular}

aTaken from Reber et al. (1996).

The numbers in the cue combination column refer from left to right to cues 1-4: A one indicates that a cue is present on a trial, while a zero indicates that a cue is absent on a trial. Frequency indicates how often a particular combination occurs out of 50 trials. Sunshine and rain indicated how often each outcome occurs. The final column indicates the probability that an outcome (sunshine in this case) will occur.

sage informing them that they were correct. In the event of an incorrect response, subjects heard a low-pitch buzzer tone and were shown a sad face as well as a text message informing them that they were incorrect and indicating the correct answer. A total of 150 trials were presented to each subject. Within each trial, a particular card configuration was presented for $5 \mathrm{sec}$ and subjects had to respond within this time. After the selection was entered, feedback was presented for 2 sec. Five self-paced introductory instruction screens appeared at the beginning of the experiment.

Following presentation of 150 trials, arousal was manipulated by having participants evaluate the intensity of their emotional response to a presented picture, i.e., the subjective correlate of peripheral physiological arousal and amygdala activity (Anderson et al. 2003) in response to the question "How intense do you feel?" The pictures were selected from the International Affective Picture Systems (IAPS) (Lang et al. 2005). A total of 75 pictures were used. In the arousal condition, $80 \%$ of the pictures were selected from those catalogued in the IAPS as having high valence (20 positive and 20 negative) and high arousal, while $20 \%$ of the pictures were neutral with low valence and low arousal. Neutral pictures were included to reduce habituation of self-reported arousal, evident in pilot data, when subjects were shown only arousing pictures. Inclusion of positive, negative, and neutral pictures was expected to limit habituation and thus evoke more consistently intense emotional responses for arousing pictures. For the neutral condition, all selected pictures were neutral in valence and low in arousal.

\section{Transfer task}

After completion of the WP task during session 1, we administered a transfer task to assess the subjects' sense of subjective probability of rain or sunshine. For session 2, the transfer task was administered after the WP task and before the picture recognition task. In either case, the test consisted of presentation of card cues that had been previously encountered in the WP task.

Single cues

Subjects were shown one of the four cues and asked the following question: "If just this card is showing, what percentage of the time will it be sunny?" This was asked for each of the four cues, and the experimenter recorded the answers on a response sheet.

Paired cues

Subjects were shown cue pairs and asked, "If these two cards are showing, what percentage of the time will it sunny?" This was asked of all six possible cue combinations. 


\section{Triple cue selection}

Subjects were shown sets of three cards and asked, "If these three cards are showing, what percentage of the time will it be sunny?" This was asked for all three possible combinations.

\section{Retention task}

To examine consolidation and retention of WP learning from the first session of training, subjects participated in a second session of another $150 \mathrm{WP}$ trials $7 \mathrm{~d}$ later.

\section{Recognition task}

Following the retention task during session 2, subjects were given a recognition task for the pictures encountered during the first session. Seventy-five pictures were shown in the recognition task, of which 50 had been previously seen in session 1, and 25 were new foil items. The 25 foil items were matched thematically with previously seen items to increase memory discrimination difficulty (10 positive, 10 negative, and five neutral). Subjects in the neutral condition were presented with 50 neutral old pictures and 25 neutral foil items.

\section{Data acquisition and analysis}

\section{Picture recognition}

The picture recognition data was analyzed for accuracy by subtracting the proportion of responses to new pictures (i.e., false alarms) from the proportion of responses to old pictures (i.e., hits).

\section{WP task}

Following prior studies, a response was scored as accurate if it was associated with the most likely outcome. Performances on trials that had a $50 \%$ chance of being rain or shine were not scored, as they did not provide any indication of learning across trials. The 150 trials were split into five blocks of 30 trials each. Session 1 and session 2 data were analyzed separately using mixedmeasures ANOVA, with trial block as the repeated-measures factor (block 1-5) and encoding condition as the between-subjects factor (neutral and arousal). Consolidation of implicit learning on the WP task across the 1-wk period separating the two WP task sessions was analyzed using a mixed ANOVA with block as the repeated-measures factor (block 5 from session 1 and block 1 from session 2) and encoding condition as the between-subjects factor (arousal vs. neutral). Further learning of the WP task during session 2 was analyzed by using the mixed ANOVA model used in session 1 .

\section{Transfer task}

The 14 different cue types were collapsed to represent five levels of cue-outcome predictability $(5 \%, 33 \%, 50 \%, 68 \%, 95 \%)$. Transfer performance during session 1 and consolidation between sessions were analyzed using a mixed-measures ANOVA with session (1 and 2) and cue-outcome probability (5\%-95\%) as the repeated-measures factors and session 1 encoding condition (arousal and neutral) as the between subjects factor.

\section{Experiment 2}

\section{Participants}

A total of 50 subjects (age range $=18-35 \mathrm{yr}$ ) participated in the study, which involved two sessions separated by $\sim 1 \mathrm{wk}$. Subjects were randomly assigned to either the arousal condition $(n=25$; five males, 20 females) or the neutral condition $(n=25$; nine males, 16 females).

\section{Materials, design, and procedure}

Analyses and procedures followed those described for Experiment 1 with the following exceptions.

\section{The WP task}

Participants performed 150 WP trials during session 1, but emotional arousal was manipulated by having participants view pic- tures (same as in Experiment 1) between every third trial (approximately every $20 \mathrm{sec}$ ).

\section{Retention task}

To examine consolidation and retention of WP learning from the first training session, subjects participated in another 150 trials of the WP task when they returned for the second session $7 \mathrm{~d}$ later. The task was the same as initial acquisition with the exception of the removal of interleaved pictures.

\section{Acknowledgments}

This research was supported by a grant from the Natural Sciences and Engineering Research Council of Canada.

\section{References}

Anderson, A.K. 2005. Affective influences on the attentional dynamics supporting awareness. J. Exp. Psychol. Gen. 134: 258-281.

Anderson, A.K., Christoff, K., Panitz, D., De Rosa, E., and Gabrieli, J.D. 2003. Neural correlates of the automatic processing of threat facial signals. J. Neurosci. 23: 5627-5633.

Anderson, A.K., Wais, P.E., and Gabrieli, J.D. 2006. Emotion enhances remembrance of neutral events past. Proc. Natl. Acad. Sci. 103: 1599-1604.

Cahill, L. 2003. Sex- and hemisphere-related influences on the neurobiology of emotionally influenced memory. Prog. Neuropsychopharmacol. Biol. Psychiatry 27: 1235-1241.

Cahill, L. and Alkire, M.T. 2003. Epinephrine enhancement of human memory consolidation: Interaction with arousal at encoding. Neurobiol. Learn. Mem. 79: 194-198.

Cahill, L. and McGaugh, J.L. 1999. Mechanisms of emotional arousal and lasting declarative memory. Trends Neurosci. 21: 294-299.

Cahill, L., Prins, B., Weber, M., and McGaugh, J.L. 1994. $\beta$-Adrenergic activation and memory for emotional events. Nature 371: 702-704.

Cahill, L., Haier, R.J., Fallon, J., Alkire, M.T., Tang, C., Keator, D., Wu, J. and McGaugh, J.L. 1996. Amygdala activity at encoding correlated with long-term, free recall of emotional information. Proc. Natl. Acad. Sci. 93: 8016-8021.

Cahill, L., Gorsky, L., and Le, K. 2003. Enhanced human memory consolidation with post-learning stress: Interaction with the degree of arousal at encoding. Learn. Mem. 10: 270-274.

Cahill, L., Uncapher, M., Kilpatrick, M., Alkire, M.T., and Turner, J. 2004. Sex-related hemispheric lateralization of amygdala function in emotionally influenced memory: An FMRI investigation. Learn. Mem. 11: $261-266$.

Canli, T., Zhao, Z, Brewer, J., Gabrieli, J.D.E, and Cahill, L. 2000. Event-related activation in the human amygdala associates with later memory for individual emotional experience. J. Neurosci. 20: RC99.

Canli, T., Desmond, J.E., Zhao, Z., and Gabrieli, J.D.E. 2002. Sex differences in the neural basis of emotional memories. Proc. Natl. Acad. Sci. 99: 10789-10794.

Cohen, N.J. and Squire, L.R. 1980. Preserved learning and retention pattern-analyzing skill in amnesia: Dissociation of knowing how and knowing that. Science 210: 207-210.

Dolcos, F., LaBar, K.S., and Cabeza, R. 2004. Interaction between the amygdala and medial temporal lobe system predicts better memory for emotional events. Neuron 42: 855-863.

Gluck, M.A., Shohamy, D., and Myers, C. 2002. How do people solve the "weather prediction" task?: Individual variability in strategies for probabilistic category learning. Learn. Mem. 9: 408-418.

Hamann, S.B., Ely, T.D., Grafton, S.T., and Kilts, C.D. 1999. Amygdala activity related to enhanced memory for pleasant and aversive stimuli. Nat. Neurosci. 2: 289-293.

Iaria, G., Petrides, M., Dagher, A., Pike, B., and Bohbot, V.D. 2003. Cognitive strategies dependent on the hippocampus and caudate nucleus in human navigation: Variability and change with practice. I. Neurosci. 23: 5945-5952.

Kensinger, E.A. and Schacter, D.L. 2005. Retrieving accurate and distorted memories: Neuroimaging evidence for effects of emotion. Neuroimage 27: 167-177.

Kilpatrick, L. and Cahill, L. 2003. Amygdala modulation of parahippocampal and frontal regions during emotionally influenced memory storage. Neuroimage 20: 2091-2099.

Kleinsmith, L.J. and Kaplan, S. 1963. Paired-associate learning as a function of arousal and interpolated interval. J. Exp. Psychol. 65: 190-193.

Knowlton, B.J., Squire, L.R., and Gluck, M.A. 1994. Probabilistic classification learning in amnesia. Learn. Mem. 1: 106-120.

Knowlton, B.J., Mangels, J.A., and Squire, L.R. 1996. A neostriatal habit learning system in humans. Science 273: 1399-1402. 
LaBar, K.S. and Phelps, E.A. 1998. Arousal-mediated memory consolidation: Role of the medial temporal lobe in humans. Psychol. Sci. 9: 490-493.

Lang, P.J., Bradley, M.M., and Cuthbert, B.N. 2005. International affective picture system (IAPS): Affective ratings of pictures and instruction manual. Technical Report A-6, University of Florida, Gainesville, FL.

Lovallo, W. 1975. The cold pressor test and autonomic function: A review and integration. Psychophysiology 12: 268-282.

MacKay, D.G., Shafto, M., Taylor, J.K., Marian, D.E., Abrams, L., and Dyer, J.R. 2004. Relations between emotion, memory, and attention: Evidence from taboo stroop, lexical decision, and immediate memory tasks. Mem. Cognit. 32: 474-488.

McDonald, R.J. and White, N.M. 1993. A triple dissociation of memory systems: Hippocampus, amygdala and dorsal striatum. Behav. Neurosci. 107: 3-22.

McGaugh, J.L. 2004. The amygdale modulates the consolidation of memories of emotionally arousing experiences. Annu. Rev. Neurosci. 27: 1-28.

McGaugh, J.L., McIntyre, C.K., and Power, A.E. 2002. Amygdala modulation of memory consolidation: Interaction with other brain systems. Neurobiol. Learn. Mem. 87: 539-552.

McIntyre, C.K., Power, A.E., Roozendaal, B., and McGaugh, J.L. 2003. Role of the basolateral amygdala in memory consolidation. Ann. N.Y. Acad. Sci. 985: 273-293.

Nielson, K.A., Radtke, R.C., and Jensen, R.A. 1996. Arousal-induced modulation of memory storage processes in humans. Neurobiol. Learn. Mem. 66: 133-142.

Nielson, K.A., Yee, D., and Erickson, K.I. 2005. Memory enhancement by a semantically unrelated emotional arousal source induced after learning. Neurobiol. Learn. Mem. 84: 49-56.

Okuda, S., Roozendaal, B., and McGaugh, J.L. 2004. Glucocorticoid effects on object recognition memory require training-associated emotional arousal. Proc. Natl. Acad. Sci. 101: 853-858.

Packard, M.G. and Cahill, L. 2001. Affective modulation of multiple memory systems. Curr. Opin. Neurobiol. 11: 752-756.

Packard, M.G., Hirsh, R., and White, N.M. 1989. Differential effects of fornix and caudate nucleus lesions on two radial maze tasks: Evidence for multiple memory systems. J. Neurosci. 9: 1465-1472.

Packard, M.G., Cahill, L., and McGaugh, J.L. 1994. Amygdala modulation of hippocampal-dependent and caudate nucleus-dependent memory processes. Proc. Natl. Acad. Sci. 91: 8477-8481.

Poldrack, R.A., Prabhakaran, V., Seger, C.A., and Gabrieli, D.E. 1999. Striatal activation during acquisition of a cognitive skill. Neuropsychology 13: 564-574.

Reber, R.J., Knowlton, B.J., and Squire, L.R. 1996. Dissociable properties of memory systems: Differences in the flexibility of declarative and nondeclarative knowledge. Behav. Neurosci. 110: 861-871.

Schacter, D.L. 1987. Implicit memory: History and current status. J. Exp. Psychol. [Hum. Learn.] 13: 412-423.

Schacter, D.L. and Tulving, E. 1994. Memory systems. MIT Press, Cambridge, MA.

Schimmack, U. and Derryberry, D. 2005. Attentional interference effects of emotional pictures: Threat, negativity, or arousal? Emotion 5: 55-66.

Walker, E.L. and Tarte, R.D. 1963. Memory storage as a function of arousal and time with homogeneous and heterogeneous lists. J. Verb. Learn. Verb. Be. 2: 113-119.

White, N.M. and McDonald, R.J. 2002. Multiple parallel memory systems in the brain of the rat. Neurobiol. Learn. Mem. 77: 125-184.

Received May 17, 2006; accepted in revised form July 10, 2006. 


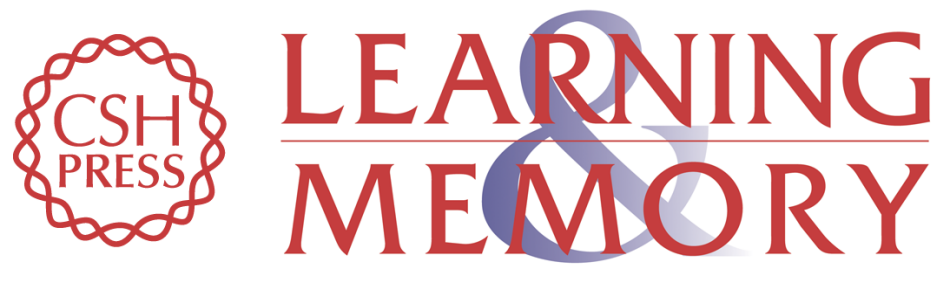

\section{Effects of emotional arousal on multiple memory systems: Evidence from declarative and procedural learning}

Stephan Steidl, Salwa Mohi-uddin and Adam K. Anderson

Learn. Mem. 2006, 13:

Access the most recent version at doi:10.1101//m.324406

References This article cites 40 articles, 14 of which can be accessed free at: http://learnmem.cshlp.org/content/13/5/650.full.html\#ref-list-1

License

Email Alerting Receive free email alerts when new articles cite this article - sign up in the box at the Service top right corner of the article or click here. 